\title{
Nucleosynthesis in Rotating massive stars and Abundances in the Early Galaxy
}

\author{
Georges Meynet ${ }^{1}$, Raphael Hirschi ${ }^{2,3}$, Sylvia Ekstrom ${ }^{1}$, André \\ Maeder $^{1}$, Cyril Georgy ${ }^{1}$, Patrick Eggenberger ${ }^{1}$, and \\ Cristina Chiappini ${ }^{1}$ \\ ${ }^{1}$ Geneva Observatory, Geneva University, \\ CH-1290 Sauverny, Switzerland \\ email: georges .meynet@unige.ch \\ ${ }^{2}$ Astrophysics group, Keele University, \\ Lennard-Jones Lab., Keele, ST5 5BG, UK \\ email: r.hirschi@epsam.keele.ac.uk \\ ${ }^{3}$ IPMU, University of Tokyo, \\ Kashiwa, Chiba 277-8582, Japan
}

\begin{abstract}
We discuss three effects of axial rotation at low metallicity. The first one is the mixing of the chemical species which is predicted to be more efficient in low metallicity environments. A consequence is the production of important quantities of primary ${ }^{14} \mathrm{~N},{ }^{13} \mathrm{C},{ }^{22} \mathrm{Ne}$ and a strong impact on the nucleosynthesis of the $s$-process elements. The second effect is a consequence of the first. Strong mixing makes possible the apparition at the surface of important quantities of CNO elements. This increases the opacity of the outer layers and may trigger important mass loss by line driven winds. The third effect is the fact that, during the main-sequence phase, stars, at very low metallicity, reach more easily than their more metal rich counterparts, the critical velocity $\dagger$. We discuss the respective importance of these three effects as a function of the metallicity. We show the consequences for the early chemical evolution of the galactic halo and for explaining the CEMP stars. We conclude that rotation is probably a key feature which contributes in an important way to shape the evolution of the first stellar generations in the Universe.
\end{abstract}

Keywords. stars: AGB, early-type, evolution, supernovae; Galaxy: halo; nucleosynthesis

\section{Introduction}

The study of the most iron poor stars in the halo offers a unique window on the nature and the evolution of the first generations of stars which provided the matter from which these halo stars were formed. Constraints on the first stellar generations may provide interesting views on topical questions as the nature of the stars which reionize the Universe, the initial mass function of the first stellar generations, the frequency of long soft Gamma Ray Bursts at very low metallicity, the timescale for the mixing of the newly synthesized products with interstellar medium material, or the conditions of star formation in the very early life of our Galaxy.

In this context, many observed features of the very iron poor stars are quite surprising and were not at all expected:

- One expected that halo stars, having a very low $[\mathrm{Fe} / \mathrm{H}]$, should show an important scatter in their abundances. Indeed, at these very early times, stars are expected to form from not well mixed clumps and thus should bear the nucleosynthetic signatures of a few peculiar events. But Cayrel et al. (2004) find that the scatter in the abundances

$\dagger$ The critical velocity is the surface equatorial velocity such that the centrifugal acceleration compensates for the local gravity. 

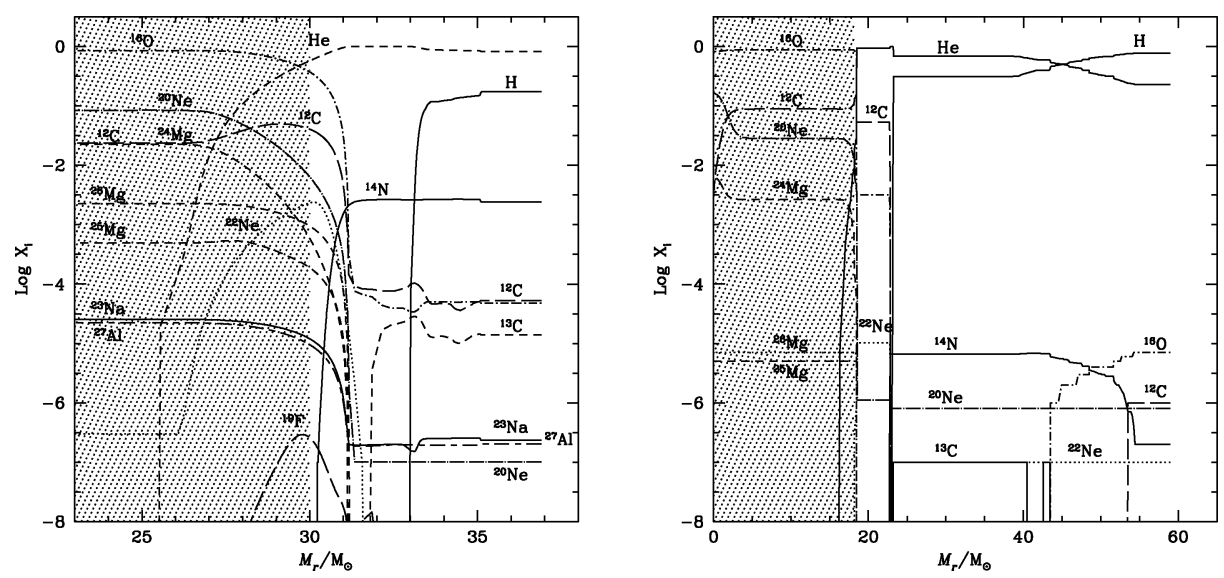

Figure 1. Left panel : variation of the abundances of various elements (in mass fraction) as a function of the Lagrangian mass in the outer layers of a $60 \mathrm{M}_{\odot}$ model at $Z=10^{-5}$ with $v_{\text {ini }}=800 \mathrm{~km} \mathrm{~s}^{-1}$ at the end of the core He-burning phase. The grey area covers the $\mathrm{CO}$ core. Right panel : same as left part for a non rotating $60 \mathrm{M}_{\odot}$ model at $Z=10^{-5}$. Models computed by Meynet \& Maeder (2002) and Meynet et al. (2006).

of several elements ratios (e.g. [ $\mathrm{Cr} / \mathrm{Fe}]$ ) is very small. It can be as low as 0.05 dex. In contrast, r-process elements and nitrogen for instance reveal large star-to-star scatters (Ryan et al. 1996; Honda et al. 2004; Andrievsky et al. 2009). Explanation are proposed in Ishimaru et al. (2004, 2006); Cescutti (2008).

- One expected that the very first generations of Pop. III stars were very massive and contributed to the enrichment of the interstellar medium through Pair Instability Supernovae (PISN, Barkat et al. 1967; Bond et al. 1984). The nucleosynthetic pattern produced by such events is well understood and presents specific features (Heger \& Woosley 2005), which were expected to be observed at least in some iron-poor halo stars. At the moment no trace of PISN has been found (Cayrel et al. 2004). Some authors have suggested an explanation why this signature has, up to now, escaped detection (Karlsson et al. 2008; Ekström et al. 2008b).

- Spectroscopic observations of halo stars (e.g. Spite et al. 2005) indicate a primary production of nitrogen over a large metallicity range at low metallicity. According to the chemical evolution models of Chiappini et al. $(2006,2008)$, primary nitrogen production by non-rotating or slow-rotating Pop III stars is not sufficient to explain the observations. For instance, primary nitrogen needs to be produced on larger ranges of masses and metallicities than expected in non rotating standard models. A similar plateau is observed in Damped Lyman Alpha (DLA) systems (Pettini et al. 2008).

- Halo stars with $\log (\mathrm{O} / \mathrm{H})+12$ inferior to about 6.5 present higher $\mathrm{C} / \mathrm{O}$ ratios than halo stars with $\log (\mathrm{O} / \mathrm{H})+12$ between 6.5 and 8.2 (Akerman et al. 2004; Spite et al. 2005). Again a similar trend is observed in DLAs (Pettini et al. 2008). This is not predicted by slow rotating models.

- The features listed above concern the bulk of the halo field stars. Now in addition to this "normal" population, there exists a group of stars showing a very different composition. These stars are collectively called Carbon-Enhanced Metal Poor stars (CEMP) and present high $[\mathrm{C} / \mathrm{Fe}]$ ratios (see the review by Beers \& Christlieb 2005). The scatter in $[\mathrm{C} / \mathrm{Fe}]$ is quite important indicating that these stars were not formed from a well mixed reservoir but acquired their peculiar high carbon abundance from locally enriched material. Some of these stars show also strong overabundances (with respect to iron) of 
Table 1. Comparison of the outputs of various stellar models.

\begin{tabular}{ccccccl}
\hline $\begin{array}{c}\text { Mass } \\
\mathrm{M}_{\odot}\end{array}$ & $Z$ & $\begin{array}{c}v_{\text {ini }} \\
\mathrm{km} \mathrm{s}^{-1}\end{array}$ & $\begin{array}{c}\Delta M_{\mathrm{MS}} \\
\mathrm{M}_{\odot}\end{array}$ & $\begin{array}{c}\Delta M_{\text {post }-\mathrm{MS}} \\
\mathrm{M}_{\odot}\end{array}$ & Max. of $\mathrm{X}_{\mathrm{s}}\left({ }^{14} \mathrm{~N}\right)$ & Reference \\
\hline 60 & 0 & 0 & 0.00 & 0.00 & 0.00 & Ekström et al. $(2008 \mathrm{a})$ \\
60 & $10^{-8}$ & 0 & 0.18 & 0.09 & $2.3410^{-10}$ & Meynet et al. $(2006)$ \\
60 & $10^{-5}$ & 0 & 0.21 & 0.22 & $2.3410^{-7}$ & Meynet et al. $(2006)$ \\
60 & $5 \times 10^{-4}$ & 0 & 0.78 & 13.29 & $2.2110^{-4}$ & Decressin et al. $(2007 \mathrm{~b})$ \\
60 & 0 & 800 & 2.32 & 2.41 & $3.5410^{-4}$ & Ekström et al. $(2008 \mathrm{a})$ \\
60 & $10^{-8}$ & 800 & 2.38 & 33.64 & $1.0210^{-2}$ & Meynet et al. $(2006)$ \\
60 & $10^{-5}$ & 800 & 6.15 & 16.57 & $2.0710^{-3}$ & Meynet et al. $(2006)$ \\
60 & $5 \times 10^{-4}$ & 800 & 20.96 & 21.79 & $3.8610^{-2}$ & Decressin et al. $(2007 \mathrm{~b})$ \\
\hline
\end{tabular}

nitrogen, oxygen and other heavy elements. At first order, these stars do appear to be formed from material having been processed mainly by $\mathrm{H}$ - and He-burning processes.

- Very interestingly, halo stars in clusters also present some surprising features. While part of the cluster population follows the same trend as the "normal" population of the field, another part presents very different chemical patterns, with for instance, strong depletions of oxygen and strong enhancements in sodium (see the review by Gratton et al. 2004). These stars do appear to be formed from material having been processed only by $\mathrm{H}$ burning.

Valid models should provide explanations for all these features. Ideally they should also provide some predictions for not yet observed characteristics. In the following we discuss the possible role of stellar axial rotation and we show how it can deeply affect the nucleosynthetic outputs of stars both in the massive $\left(>8 \mathrm{M}_{\odot}\right)$ and intermediate mass range $\left(2<\mathrm{M} / \mathrm{M}_{\odot}<8\right)$ and how it can explain some of the above observed features.

\section{Effects induced by rotation}

The physics of rotation included in present stellar models has been recently exposed in Maeder (2009). Here we just briefly recall the main effects. First rotation triggers many instabilities in stellar interiors. These instabilities participate in the transport of chemical species and of angular momentum in, otherwise, stable, radiative regions.

Around solar $Z$, rotating models improve a lot the agreements between the predictions of the stellar models and the observations. For instance the changes of the surface chemical abundances can be reproduced when the effects of rotation are accounted for (Maeder et al. 2009). Also the variation with the metallicity of the number ratio of Wolf-rayet to O-type stars can be reproduced (Meynet \& Maeder 2005). When the same physics, allowing these successes at near solar metallicity, is implemented in stellar models at very low metallicity, one notes interesting consequences. The most important consequence (the other consequences can actually be deduced from this one) is that the stars with the lowest metallicity (other characteristics being kept the same) will be more mixed by rotational mixing than the stars with a higher metal content (Maeder \& Meynet 2001). This comes mainly from the fact that at low $Z$, stars are more compact. This makes the gradient of the angular velocity inside the star steeper and thus the transport of the chemical species by shear mixing more efficient. From this characteristic, occurring at low $Z$, two consequences of great interest for the questions we are discussing here have been found: first rotating massive and intermediate mass stars can produce important amounts of primary nitrogen. The level of production depends on the initial velocity of the models. Other elements have their production boosted by rotational mixing, among 
them are ${ }^{13} \mathrm{C},{ }^{22} \mathrm{Ne} \dagger$. This is shown in Fig. 1, where the compositions of a non-rotating and a rotating $60 \mathrm{M}_{\odot}$ model at $Z=10^{-5}$ are plotted.

A second consequence is that, during the core He-burning phase, the surface of the star can be strongly enriched in CNO elements, increasing its surface metallicity by many orders of magnitudes (Meynet \& Maeder 2002; Hirschi 2007). This strong surface metallicity enhancement can trigger important mass losses driven by radiatively line driven winds. The material ejected in that way will bear the nucleosynthetic signature of both H- and He-burning processes.

Another effect which occurs preferentially at low $Z$ is the loss of mass through mechanical mass loss. What we call here mechanical mass loss is the loss of mass when the surface velocity is so high that, at the equator, the centrifugal acceleration is equal to the gravity. When such circumstances are realized, a tiny kick suffices to launch matter into a keplerian orbit around the star and thus to form an equatorial disk. What does happen to that material remains quite speculative. A reasonable hypothesis is that this material is lost. This process does occur in near solar metallicity for sufficiently rapidly rotating B-type stars. Not for O-type stars, because, at solar metallicity, they lose too much mass and therefore angular momentum by stellar winds (Ekström et al. 2008c). At very low metallicity, one expects that the reaching of the critical limit will also occur for more massive stars as O-type stars, because, in contrast to what happens at high metallicities, radiatively driven winds are weaker during the MS phase and thus do not remove the angular momentum brought to the surface by the meridional currents.

What is the importance of these various effects? How do they vary as a function of the initial mass, metallicity and initial rotational velocities? Some elements of response can be obtained from Table 1 . We can note the following trends

- The mass lost during the MS phase $\left(\Delta M_{\mathrm{MS}}\right)$, in rotating models, is due, at these very low metallicities, mainly to the reaching of the critical limit (see column 4 of Table 1). The effect of mechanical mass loss can be estimated by comparing $\Delta M_{\mathrm{MS}}$ for the rotating and the non-rotating models. We see that the mechanical mass loss has a kind of metallicity dependence. The model at higher metallicity loses much more mass by this process than models at lower metallicities. The reason for this is due to three facts: first, a given value of the velocity on the ZAMS corresponds, at high $Z$, to a higher value of the ratio $v / v_{\text {crit }}$ than at low $Z$ (see Fig. 12 in Ekström et al. 2008c). Second, the meridional currents slow down the internal regions and accelerate the surface and are thus the main agents which bring the velocity of the surface near the critical one. Third, these currents are slower in more compact stars. Therefore, in low metallicity stars, it takes more time, starting from a given initial velocity, to reach the critical limit $\ddagger$. When the critical limit is reached, the surface velocity remains near the critical value until the end of the MS phase. This is due to the following behaviour: the star encounters the critical limit for the first time, mass is lost at the equator, angular momentum is removed, the star evolves away from the critical limit. Then meridional currents bring again angular momentum to the surface making the star to evolve back against the critical limit. The cycle begins again. The timescale for the evolution back to the critical limit will be shorter at higher than at lower metallicities for the same reason as above, and thus this will favor higher mass losses by this process. The material released by this mechanical

$\dagger$ Enhancement in the abundance of ${ }^{22} \mathrm{Ne}$ in the He-core of the rotating model has a strong impact on the $s$-process according to the work by Pignatari et al. (2008).

$\ddagger$ Let us note that, due to these three effects, without mass loss, stars would reach the critical limit more rapidly at high metallicity. However, when the metallicity is near the solar metallicity, the strong line driven stellar winds remove rapidly the angular momentum brought to the surface by the meridional currents and prevent the high mass losing stars to reach the critical limit. 
mass loss is only enriched in H-burning products, since it is ejected during the MS phase. Decressin et al. $(2007 \mathrm{~b}, \mathrm{a})$ have studied the consequences of such models for explaining the chemically peculiar stars observed in globular clusters.

- In the models at $Z$ equal to $10^{-8}$ and $10^{-5}$, a phase exists where the star is in the red part of the HR diagram, and presents strong enhancements of its surface CNO content. The last column of Table 1 indicates the maximum value of the abundance of ${ }^{14} \mathrm{~N}$ reached at the surface. In non-rotating models, no change of the surface abundance occurs for $Z \leqslant 10^{-5}$. In the non-rotating $Z=5 \times 10^{-4}$ model, nitrogen enhancement occurs as a result of mass loss. Only secondary nitrogen is produced in non metal-free, non-rotating models. In the rotating models, primary nitrogen is produced. The most efficient producers are models with $Z=10^{-8}$ and $10^{-5}$. In these models, the apparition at the surface of primary CNO elements triggers high mass loss rates (see column 5 in Table 1). The material ejected in that way has been processed by both $\mathrm{H}$ - and He-burning processes (Meynet et al. 2006; Hirschi 2007). For the velocities and mass considered here, this only occurs for non-zero very low metallicity, but not for Pop III stars. At near solar metallicities, this does not occur for models with the same initial angular momentum content. Why? For $Z=0$, we shall see below that the mixing during the core He-burning phase is less efficient than in non-zero metallicity stellar models. At higher metallicities, rotational mixing is less efficient. Furthermore the line driven winds become more important, and in very massive stars, they may remove the H-burning regions early during the core He-burning phase preventing primary nitrogen production. In the following, we argue that the material ejected under the form of this wind triggered by the self enrichment of the surface, presents strong similarities with the abundance pattern observed at the surface of the CEMP stars.

- The rotating models produce primary nitrogen (and also primary ${ }^{13} \mathrm{C}$ and ${ }^{22} \mathrm{Ne}$ ). The most efficient producers are the models with $Z$ equal to $10^{-8}$ and $10^{-5}$. The pop III stars produce less primary nitrogen for the following reason: due to the absence of CNO elements, these models begin to convert $\mathrm{H}$ into He through the pp chains. The energy output from these chains is not sufficient to compensate for the high luminosity of the star. Therefore the rest of the energy has to be extracted from the gravitational reservoir and the star must contract. Contraction occurs until the central temperatures reach sufficient high values to activate the triple alpha reaction. Some carbon is then produced and the CNO cycle can be activated. From this stage on, the H-burning is pursued as in more metal rich stars, through the CNO cycle. A consequence of this is that the core H-burning occurs at temperatures very similar to that of the core He-burning. Thus, in Pop III stars, at the end of the core H-burning phase, the core does not need to contract a lot in order to re-activate the He-burning reactions. This absence of strong contraction maintains the star in the blue part of the HR diagram during most of the core He-burning phase. It also prevents a strong gradient of angular velocity to form at the border of the core and therefore efficient shear mixing. Less primary nitrogen is formed. In more metal rich models, less primary nitrogen is produced both because mixing is less efficient and because the H-burning shell is more distant from the He-burning core.

\section{Nucleosynthesis from "spinstars"}

Let us call spinstars, stars whose evolution or nucleosynthesis is deeply affected by axial rotation. Such spinstars can contribute in two ways in shaping the chemical composition of the halo stars that we observe today. First the ejecta of stars of different masses, metallicities and initial rotation velocities can be mixed with ISM and provide the raw material from which the "chemically normal" halo population stars are formed. 

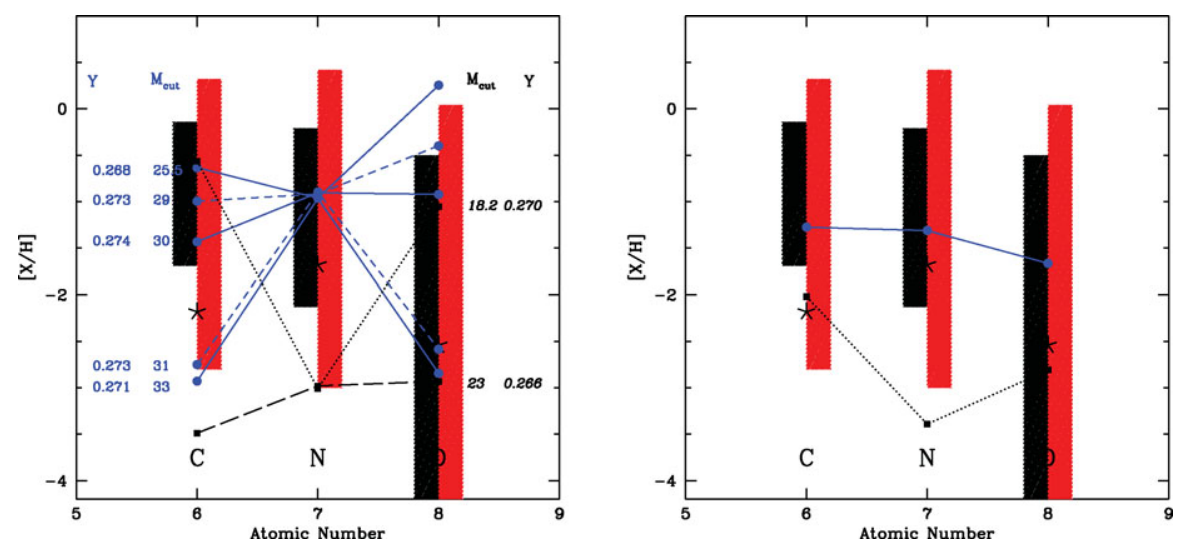

Figure 2. Left panel: Composition of the mixture composed of wind material of $60 \mathrm{M}_{\odot}$ models at $Z=10^{-5}$ and of layers ejected by the supernova diluted with 10 times more interstellar material. The different curves correspond to the model WIND+SUPERNOVA in Table 2, various values of the mass-cut are considered. The continuous and short dashed lines curves correspond to the rotating model with $v_{\text {ini }}=800 \mathrm{~km} \mathrm{~s}^{-1}$. The dotted lines and long dashed curves curves correspond to the non-rotating models (models from Meynet \& Maeder 2002). As the mass cut increases (downwards), the amount of carbon and oxygen ejected by the supernova decreases. The stars show the observed values for the most iron poor star known today (Frebel et al. 2008). The vertical hatched zones show the range of observed values for CEMP-no stars with $\log g$ superior to 3.8 (left grey zone, 5 stars in the sample) and with $\log g$ inferior to 3.8 (right red zone, 20 stars in the sample). The values were taken from Tables 1 and 2 of Masseron et al. (2009). Only upper limits are given for $[\mathrm{O} / \mathrm{H}]$, this is why the columns extend down to the bottom of the figure. Right panel : The continuous line shows the composition obtained by mixing the outer envelope (mass above the $\mathrm{CO}$ core) at the E-AGB phase of our rotating $7 \mathrm{M}_{\odot}$ stellar model with 100 times more of interstellar medium. The dotted curve with squares shows the composition obtained in the same way (mass above the $\mathrm{CO}$ core and interstellar medium) using our analog non-rotating $7 \mathrm{M}_{\odot}$ stellar model.

In order to compute the evolution with time or with $[\mathrm{Fe} / \mathrm{H}]$ of such stars, detailed chemical evolution models have to be used. The models by Chiappini et al. (2006) show that the N/O plateau observed at low $Z$ can be well reproduced when yields of rotating models $\left(V_{\mathrm{ini}}=800 \mathrm{~km} \mathrm{~s}^{-1}\right)$ are used. The slower rotating models can produce a plateau for the nitrogen to oxygen ratio at low metallicities but the level is two to three orders of magnitude below the observed one. Non-rotating models would still be much lower and would not produce a plateau at low $Z$. (see Fig. 4 Chiappini et al. 2006). Not only the N/O ratio is very well reproduced by these models but also the $\mathrm{C} / \mathrm{O}$ upturn mentionned in the introduction. It would be extremely interesting to obtain information of the ${ }^{12} \mathrm{C} /{ }^{13} \mathrm{C}$ ratio in very metal poor stars, whose surface still reflects the abundances of the cloud from which these stars were formed. Chiappini et al. (2008) show that the slow and fast rotating models predict very different values for this ratio.

Stars can also form from interstellar material enriched by one or a very small number of nucleosynthetic events. In that case one expects some important scatter from one star to another and also some strong differences with respect to stars formed from the well mixed reservoir. The CEMP stars are likely formed in that way.

We show in Table 2 the chemical composition of various components (winds, wind plus supernova ejecta, envelope of an early-AGB star) of material ejected by various stellar models. The composition indicated in that table would be the one of stars formed from pure ejecta. Looking at the results of Table 2, the following trends can be deduced: 
Table 2. Chemical composition of the ejecta of various models in mass fraction. The number ratio ${ }^{12} \mathrm{C} /{ }^{13} \mathrm{C}$ is also indicated.

\begin{tabular}{|c|c|c|c|c|c|c|c|c|c|}
\hline $\mathrm{M}_{\mathrm{ini}}$ & $\mathrm{Z}_{\text {in i }}$ & $v_{\text {in i }}$ & $M_{\text {eje }}$ & $\mathrm{X}_{\mathrm{H}}$ & $\mathrm{X}_{\mathrm{He}}$ & $\mathrm{X}_{\mathrm{C} 12}$ & ${ }^{12} \mathrm{C} /{ }^{13} \mathrm{C}$ & $\mathrm{X}_{\mathrm{N} 14}$ & $\mathrm{X}_{\mathrm{O} 16}$ \\
\hline \multicolumn{10}{|c|}{ WIND } \\
\hline 85 & $10^{-8}$ & 800 & 65.2 & 0.27 & 0.55 & $1.0 \mathrm{e}-01$ & 11.4 & $3.0 \mathrm{e}-02$ & $5.0 \mathrm{e}-02$ \\
\hline 60 & $10^{-8}$ & 800 & 36.2 & 0.40 & 0.59 & $1.3 \mathrm{e}-04$ & 5.4 & $5.5 \mathrm{e}-03$ & $1.7 \mathrm{e}-04$ \\
\hline 40 & $10^{-8}$ & 700 & 4.2 & 0.68 & 0.32 & $1.9 \mathrm{e}-03$ & 6.8 & $8.6 \mathrm{e}-04$ & $5.8 \mathrm{e}-04$ \\
\hline 60 & $10^{-5}$ & 800 & 23.1 & 0.47 & 0.53 & $1.4 \mathrm{e}-04$ & 4.6 & $4.2 \mathrm{e}-04$ & $1.2 \mathrm{e}-05$ \\
\hline \multicolumn{10}{|c|}{ WIND+SUPERNOVA (different mass cuts) } \\
\hline 60 & $10^{-5}$ & 0 & 37 & 0.48 & 0.52 & $1.7 \mathrm{e}-07$ & $3.65 \mathrm{e}+00$ & $4.7 \mathrm{e}-06$ & $2.1 \mathrm{e}-06$ \\
\hline 60 & $10^{-5}$ & 0 & 41.8 & 0.42 & 0.57 & $6.4 \mathrm{e}-03$ & $1.55 \mathrm{e}+05$ & $4.3 \mathrm{e}-06$ & $5.1 \mathrm{e}-03$ \\
\hline 60 & $10^{-5}$ & 800 & 27 & 0.42 & 0.58 & $2.0 \mathrm{e}-05$ & $4.70 \mathrm{e}+00$ & $7.3 \mathrm{e}-04$ & $1.7 \mathrm{e}-05$ \\
\hline 60 & $10^{-5}$ & 800 & 29 & 0.39 & 0.61 & $3.4 \mathrm{e}-05$ & $7.32 \mathrm{e}+00$ & $8.5 \mathrm{e}-04$ & $8.5 \mathrm{e}-05$ \\
\hline 60 & $10^{-5}$ & 800 & 30 & 0.38 & 0.61 & $8.6 \mathrm{e}-04$ & $1.90 \mathrm{e}+02$ & $8.4 \mathrm{e}-04$ & $6.9 \mathrm{e}-03$ \\
\hline 60 & $10^{-5}$ & 800 & 32 & 0.37 & 0.60 & $2.4 \mathrm{e}-03$ & $5.39 \mathrm{e}+02$ & $8.1 \mathrm{e}-04$ & $2.3 \mathrm{e}-02$ \\
\hline 60 & $10^{-5}$ & 800 & 34.5 & 0.33 & 0.55 & $5.4 \mathrm{e}-03$ & $1.37 \mathrm{e}+03$ & $7.3 \mathrm{e}-04$ & $1.0 \mathrm{e}-01$ \\
\hline \multicolumn{10}{|c|}{ E-AGB ENVELOPE } \\
\hline 7 & $10^{-5}$ & 0 & 6.1 & 0.65 & 0.34 & $2.1 \mathrm{e}-03$ & $4.30 \mathrm{e}+05$ & $2.9 \mathrm{e}-06$ & $2.0 \mathrm{e}-04$ \\
\hline 7 & $10^{-5}$ & 800 & 5.7 & 0.63 & 0.35 & $9.6 \mathrm{e}-03$ & $1.04 \mathrm{e}+02$ & $3.2 \mathrm{e}-03$ & $7.8 \mathrm{e}-03$ \\
\hline
\end{tabular}

- Stars made of pure ejecta (or diluted with small amount of interstellar material) would be He-rich. This is true whatever the source of the ejecta, rotating or non-rotating, wind material, wind plus supernova material, or envelope of an early AGB star. Such stars would be depleted in lithium.

- Low values for the ${ }^{12} \mathrm{C} /{ }^{13} \mathrm{C}$ ratio indicates that the ejecta are rich in $\mathrm{CNO}$-processed material. This conclusion does not depend on the degree of dilution with interstellar material in case we consider ejecta of spinstar models. Indeed. in rotating models, both ${ }^{12} \mathrm{C}$ and ${ }^{13} \mathrm{C}$ are produced through primary channels in so large quantities that the dilution should be enormous for changing it.

- We see that, when the contribution of the supernova increases, the ${ }^{12} \mathrm{C} /{ }^{13} \mathrm{C}$ ratio becomes larger. Also the abundances of carbon and oxygen increase. This is because some layers, rich in He-burning products, are ejected. We conclude that stars presenting high $[\mathrm{N} / \mathrm{C}]$ and $[\mathrm{N} / \mathrm{O}]$ ratios cannot be mainly made from material processed by He burning and thus from supernova ejecta (except if the supernova only eject the outermost layers). This is also independent of the degree of dilution with interstellar material. It is also independent of the models considered (rotating or non rotating).

- We see that rotating models are the only ones, among those presented in Table 2, showing strong enhancements of the three CNO elements simultaneously. The reason for this is that, in these models, mixing of He-burning products with H-burning products occurred in the star which has ejected the material.

Comparisons with observed values are made in Fig. 2. Composition of pure ejecta mixed with some amount of interstellar material are plotted. An important point to mention in order to correctly interpret this figure is the following: for the rotating models, the positions of the different curves in this diagram do not much depend on the initial metallicity. Here the quantities are plotted for models at $Z=10^{-5}$. Would we have plotted data obtained from the rotating model at $Z=10^{-8}$, similar results would have been obtained. This is because all the three isotopes (and also ${ }^{13} \mathrm{C}$ ) are produced by (quasi) metallicity independent channels. For the non-rotating models, we have a different situation. While ${ }^{12} \mathrm{C}$ and ${ }^{16} \mathrm{O}$ have a strong primary component, ${ }^{14} \mathrm{~N}$ and ${ }^{13} \mathrm{C}$ are only secondary. This 
means that, would we have plotted the results for a model with $Z=10^{-8}$ for instance, the point corresponding to ${ }^{14} \mathrm{~N}$ would have shifted downwards by about 3 dex! Keeping this in mind, we see that that non-rotating massive stars cannot fit the observed values of the Frebel star for instance $\left(Z \sim 10^{-6}\right)$. In contrast, rotating models produce situations where the three elements are enhanced. This is true for massive stars but also for intermediate mass stars. We also see that with a dilution factor of 10, CEMP stars made up of winds and of a small amounts of supernova ejecta would be He-rich. The ${ }^{12} \mathrm{C} /{ }^{13} \mathrm{C}$ ratios would be as indicated in Table 2 (the mass ejected is equal to 60 minus $M_{\text {cut }}$ ). Greater dilutions factors (about 10 times higher) seem to be required in case CEMP stars result from the mixture of an E-AGB envelope with interstellar material. In that case, these stars would not be He-rich. They would also show high lithium abundances at least equal to the Spite Li plateau in case the depletion process in those very metal poor stars occurred as it occurred in the stars of the Spite plateau. The ${ }^{12} \mathrm{C} /{ }^{13} \mathrm{C}$ ratios would be equal to 105 and 2560 for the rotating and non-rotating case respectively.

\section{References}

Akerman, C. J., Carigi, L., Nissen, P. E., Pettini, M., \& Asplund, M. 2004, A\&̈A, 414, 931

Andrievsky, S. M., Spite, M., Korotin, S. A., et al. 2009, AESA, 494, 1083

Barkat, Z., Rakavy, G., \& Sack, N. 1967, Physical Review Letters, 18, 379

Beers, T. C. \& Christlieb, N. 2005, ARA\&AA, 43, 531

Bond, J. R., Arnett, W. D., \& Carr, B. J. 1984, ApJ, 280, 825

Cayrel, R., Depagne, E., Spite, M., et al. 2004, A\&A, 416, 1117

Cescutti, G. 2008, A\&SA, 481, 691

Chiappini, C., Ekström, S., Meynet, G., et al. 2008, A\&A, 479, L9

Chiappini, C., Hirschi, R., Meynet, G., et al. 2006, A\&A, 449, L27

Decressin, T., Charbonnel, C., \& Meynet, G. 2007a, A\&A, 475, 859

Decressin, T., Meynet, G., Charbonnel, C., Prantzos, N., \& Ekström, S. 2007b, A\&A, 464, 1029

Ekström, S., Meynet, G., Chiappini, C., Hirschi, R., \& Maeder, A. 2008a, A\&A, 489, 685

Ekström, S., Meynet, G., \& Maeder, A. 2008b, in IAU Symposium, Vol. 250, IAU Symposium, ed. F. Bresolin, P. A. Crowther, \& J. Puls, 209-216

Ekström, S., Meynet, G., Maeder, A., \& Barblan, F. 2008c, A\&AA, 478, 467

Gratton, R., Sneden, C., \& Carretta, E. 2004, ARA\&A, 42, 385

Heger, A. \& Woosley, S. 2005, in IAU Symposium, Vol. 228, From Lithium to Uranium: Elemental Tracers of Early Cosmic Evolution, ed. V. Hill, P. François, \& F. Primas, 297-302

Hirschi, R. 2007, A\& $A, 461,571$

Honda, S., Aoki, W., Kajino, T., et al. 2004, ApJ, 607, 474

Ishimaru, Y., Wanajo, S., Aoki, W., \& Ryan, S. G. 2004, ApJ, 600, L47

Ishimaru, Y., Wanajo, S., \& Prantzos, N. 2006, in International Symposium on Nuclear Astrophysics - Nuclei in the Cosmos

Karlsson, T., Johnson, J. L., \& Bromm, V. 2008, ApJ, 679, 6

Maeder, A. 2009, Physics, Formation and Evolution of Rotating Stars, ed. A. Maeder

Maeder, A. \& Meynet, G. 2001, A\& $A, 373,555$

Maeder, A., Meynet, G., Ekström, S., \& Georgy, C. 2009, Communications in Asteroseismology, 158,72

Masseron, T., Johnson, J. A., Plez, B., et al. 2009, ArXiv e-prints

Meynet, G., Ekström, S., \& Maeder, A. 2006, A\&A, 447, 623

Meynet, G. \& Maeder, A. 2002, A\& A, 390, 561

Meynet, G. \& Maeder, A. 2005, A\&A, 429, 581, paperXI

Pettini, M., Zych, B. J., Steidel, C. C., \& Chaffee, F. H. 2008, MNRAS, 385, 2011

Pignatari, M., Gallino, R., Meynet, G., et al. 2008, ApJ, 687, L95

Ryan, S. G., Norris, J. E., \& Beers, T. C. 1996, ApJ, 471, 254

Spite, M., Cayrel, R., Plez, B., et al. 2005, A\& A, 430, 655 